\title{
Extraordinary Dynamic Near Vision in Champion Shotgun Shooters: PDI Check Evaluation of Stereo and Color without Floor Effect
}

This article was published in the following Dove Press journal: Clinical Ophthalmology

\author{
Steven Brown (iD) \\ Clayton Rue ${ }^{2}$ \\ Kyle Smith $\mathbb{1 D}^{3}$ \\ Robert Arnold (iD) ${ }^{4}$
}

'Martin Methodist University, Pulaski, TN, USA; ${ }^{2}$ NSCA Level III Instructor, Anchorage, AK, USA; ${ }^{3}$ Accurate Vision, Anchorage, AK, USA; ${ }^{4}$ Alaska Blind Child Discovery, Alaska Children's EYE \& Strabismus, Anchorage, AK, USA

\section{Video abstract}

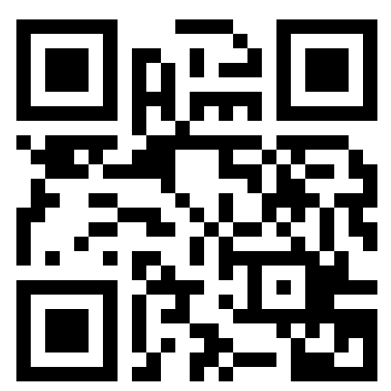

Point your SmartPhone at the code above. If you have a QR code reader the video abstract will appear. Or use: https://youtu.be/dxZFZu7g8Aw
Correspondence: Robert Arnold

The Alaska Blind Child Discovery, Alaska Children's EYE \& Strabismus, 3500

Latouche \#280, Anchorage, AK, 99508,

USA

Tel +I (907)56I-1917

$\mathrm{Fax}+\mathrm{I}(907) 563-5373$

Email eyedoc@alaska.net
Background: Conventional near and stereo tests have a "floor effect;" inability to discriminate normal from super-normal. Shotgun champions require unique performance in binocularity and rapid color discrimination.

Patients and Methods: Twenty-nine champion clay-target shooters, 14 normal Army and 12 color-normal subjects were tested with conventional near vision tests and on PDI-Check, a series of dynamic, near vision tests on the autostereoscopic Nintendo console.

Results: Conventional tested shotgunners had all perfect Ishihara and $75 \%$ best $40 \mathrm{sec}$ Stereo fly while Army had 92\%perfect Ishihara and 69\% best stereo fly. On PDI-Check, experienced shotgunners with median stereo of 17 (IQR 12-41) arc sec were better than Army volunteers 59 (IQR 30 to 487) arc seconds (Mann-Whitney U=62, p=0.04). Median PDI-Check for shotgun compared to Army was $88 \%$ compared to $84 \%$ for blue cone, $90 \%$ compared to $84 \%$ for green and $77 \%$ compared to $72 \%$ (red, MannWhitney $\mathrm{p}=0.05$ ). Adding dark cherry filter shooting glasses improved the red and green color detection $(\mathrm{p}<0.01)$. This study lacked power to detect eye dominance using PDI Check color or stereo.

Conclusion: Many champion shotgun shooters had outstanding dynamic near stereo and better-than-average color vision which could be optimized with dark filter spectacles for red and green hues. PDI Check not only helps identify abnormal binocularity and color vision, but it can clarify supranormal vision as well.

Keywords: stereopsis, eye dominance, color vision

\section{Plain Language Summary}

Champion adult and collegiate shotgun shooters must see fast-moving colored clay pigeons to align their gun, pull the trigger and then score highly. To determine if exceptional vision could part of their advantage, we tested near visual acuity, stereo and red-green-blue color on a dynamic Nintendo 3DS autostereoscopic vision screening game called PDI Check. The median stereo score on PDI Check for shotgun shooters was 17 seconds of arc compared to 59 seconds of arc for normal-sighted, Army volunteers. The shotgun shooter's combined color score of $86.6 \%$ was better than the mean score of $81.8 \%$ for color-normal, age-matched subjects. Indeed, the binocular performance and aspects of color vision, especially with shooting goggles, were particularly good in shotgun champions compared to age-matched, normal subjects. PDI check on the sturdy, portable 3-dimensional game console is useful for screening patients with vision defects, but it can also identify individuals with extraordinarily keen vision. 


\section{Introduction}

Vision is required to perform at an ordinary level at many tasks. Exceptional vision is required to excel at certain tasks such as shooting and other sports. The components of excellent vision can be separated into right and left visual acuity, stereopsis and color discrimination. Separating exceptional from ordinary requires a testing tool without a "floor effect."1

Certain conventional tests can identify individuals whose vision is in a normal range like 6/6 (20/20) "normal" visual acuity. In a classroom of normal, straight-eyed high school girls, almost all would score the very best 40 seconds of arc stereo on the Titmus Fly. ${ }^{2}$ Since "colorblindness" is mainly inherited by boys, almost all the girls also might score 14 out of 14 Ishihara color plates. Since each of these conventional tests, and a 20/20 visual acuity chart have a measurement "floor;" their thresholds in detection are incapable of quantifying supra-threshold, excellent vision, from "normal" vision. ${ }^{3}$

Shotgun, or clay-target shooting, is a sport requiring rapid peripheral detection and hand-eye pursuit of a fastflying, colored clay pigeon. ${ }^{4}$ Special formats of peripheral location and distance differentiate skeet, trap and sporting clay events. The score in Olympic, international, collegiate and regional competitions is dependant how many pigeons are struck or broken. To improve preception of the colored target, many shooters use expensive filtered goggles. Expert clay pigeon shooters produce consistent scores near $100 \%$ hits in part due to excellent binocular and color vision.

We undertook this study using a novel, dynamic near vision application $^{5,6}$ on a Nintendo 3 DS console to characterize the quality of vision in champion shotgun shooters in comparison with groups of age-matched, normal subjects.

\section{Methods}

This study has institutional Review Board approval from Pacific University [1098158-1] and is compliant with the Health Information Portability and Accountability Act and the Declaration of Helsinki. Subjects and/or their legal guardians signed written informed consent and/or assent. De-identified data can be accessed from http://www.abcdvision.org/references/PDI\%20shotgun\%200.2.15.pdf

Conventional testing included Rosenbaum near visual acuity, Ishihara concise color plates and Titmus Stereo fly at a distance of $30 \mathrm{~cm}$ with best refractive correction.

\section{Subjects}

Experienced and expert competitive shotgun shooters regardless of prior eye history or spectacle use comprised the experimental group. The control groups were taken from previous PDI Check validation using identical component versions of the app. One group of controls came from healthy Army volunteers with no prior history of eye disease $^{7}$ and the other group was asymptomatic control subjects when PDI Check color test was applied to other patients identified as inherited color deficient. ${ }^{7}$

\section{Testing Environment}

Each subject was tested indoors with normal ambient light without glare from the two Nintendo 3DS XL screens. Each subject was tested in their conventional condition whether they wore optically correcting lenses. Each shotgun shooter was also tested with colored filtering goggles. The selected shooting lenses were 76 High Contrast "filter" (yellow) and 18 Red High Contrast "dark" (cherry made by Pilla Sport, Ridgefield, Connecticut)

\section{Vision Testing App and Device}

PDI Check presents a dynamic, forced-choice game on the autostereoscopic screen of the Nintendo 3DS XL game console. PDI check is capable of presenting fine to gross stereopsis and monocular Landholt-C optotype rivalry acuity and monocular color rivalry testing because a different set of pixel pairs is presented to right and left eyes through the autostereoscopic, barrier upper screen while the subject indicates as soon as one random quadrant is perceived as different from the other three quadrant presentations. The color vision presents an iso-luminance gray background onto which one quadrant disk initially the same gray, but evolving into more-and-more vibrant teal, pink or purple colors helpful for identifying protanopia, deuteranopia or tritanopia. Each subject was not allowed to practice with the PDI Check device, but was scored on their initial exposure to the battery of stereo, monocular near dynamic acuity and color tests. PDI Check testing used a standard Nintendo 3DS XL console development kit with version 0.2.15. A 2-dimensional example of the game is shown: https://vimeo.com/pdimd/shotgunpdi

\section{Statistical Methods}

Sample size: given a mean of 50, a standard deviation of 150 , and an alpha of 0.05 and a power of 0.80 , the sample size needed to detect a difference of 150 is 16 . 
Normality of data distributions was tested with kurtosis and skewness. Age for shotgun and control groups was normally distributed so parametric analyses were done. Non-parametric data pairs were compared with MannWhitney test while the Kruskal-Wallis test was used to compare three or more groups. A p-value less than 0.05 was considered significant.

\section{Results}

Twenty-nine shotgun shooters completed PDI Check dynamic forced-choice near vision testing compared to conventional stereopsis and isochromatic plate testing. Ages ranged from 14 to 74 years with 12 teen aged, 11 in their twenties and four older than 30 years. 14 were considered experts, 11 had advanced experience and 4 were novices. The expert and advanced were analyzed together as 25 "experienced" shotgun shooters. Ten were wearing corrective lenses. Almost all described themselves as right eye dominant except four who claimed they were left eye dominant and one was "middle eye" dominant. ${ }^{8}$ Eleven had scored a perfect 100 in either skeet, trap and or sporting clays competitions while another eight scored between 93 and 99 clay pigeons broken out of 100 .

Two control groups were tested on different visual aspects of the PDI Check app. For a control group relative to PDI check dynamic stereo and monocular acuity, data from a group of 14 healthy Army volunteers aged 21 to 36 years were used: two of these were found to show Titmus stereo less than 150 arc seconds. The mean age of the shotgunners $(24 \pm 6$ years) did not differ from the Army volunteers $(29 \pm 4$ years; $t$-test $\mathrm{t}(41)=1.2, \mathrm{p}=0.25)$. The control group for PDI Check monocular color testing was a group of 12 normal subjects aged 8 to 61 years who had normal red, green and blue color on Innova Rabin testing. The mean age of the color normals ( $31 \pm 9$ years) did not differ from the shotgunners $(\mathrm{t}=1.3, \mathrm{p}=0.19)$.

Table 1 shows median, interquartile range, 95 percentiles and mean and standard deviation for stereo testing, near right and left acuity testing and right and left dynamic color testing for red-cone, green-cone and blue cone perception. Figure 1 shows shotgun versus Army near vision performance on stereo and visual acuity. Also shown are age-matched normals who all passed Innova Rabin color and the shotgunners performing trichromatic color highlighting the impact of two types of filtered spectacles. For the shotgun shooters, PDI Check stereo had kurtosis 7.7 and skewness 2.5. Right and left near visual acuity had kurtosis 18.2 and 17.3, respectively, and skewness 4.2 and 3.9. Kurtosis for red, green and blue color right eye was $-0.75,2.1$ and 2.9 while skewness was $-0.43,-1.6$ and -1.7 . Since these variables are not normally distributed, non-parametric statistics are employed.

Significant differences between the experienced shotgunners and the Army volunteers did not occur for PDI Check near right eye or left eye acuity, or for Titmus stereo, but did for PDI Check stereo with arc seconds median 17 (IQR 12-41) compared to Army 59 (IQR 30487) (Mann-Whitney $\mathrm{U}(14,25)=77, \mathrm{p}<0.01$ ) and for $\log _{10}$ stereo (Mann-Whitney $\mathrm{U}(14,29)=95, \mathrm{p}<0.01$ ). Notice that the Army subjects had some with stereo worse than 100 arc seconds, but there were many more shotgun shooters scoring exceptionally good stereo acuity.

For the right eye, the shotgunners and the color normals did not differ except a suggestion of a difference for red cone (Mann-Whitney $\mathrm{U}(12,29)=107, \mathrm{p}=0.05$ ). Using an averaged score for all three colors right and left, the shotgunners median $86.6 \%$ (IQR $81.2,88$ ) outscored the median $81.8 \%$ (IQR 76.4, 84.8) for the normal subjects (Mann-Whitney $\mathrm{U}(12,29)=105, \mathrm{p}=0.05$ ). The perceptionimproving impact of color filters for right eye was significant with dark cherry filter for red (Mann-Whitney U $(27,29)=88, p<0.01)$ and dark cherry filter for green (Mann-Whitney $\mathrm{U}(27,29)=160, \mathrm{p}<0.01$ ).

With normal subjects combined with experienced shotgun shooters, there was a positive correlation between Stereo Fly (F) and PDI Check stereo (P); F $=0.51(\mathrm{P})+$ $37, \mathrm{r}(41)=0.6, \mathrm{p}<0.01$.

An attempt was made to predict shooting eye dominance ( +1 right eye, -1 left eye and 0 "middle eye") from the autostereoscopic stereo and monocular testing scores from PDI Check in experienced shotgun shooters. For shooters with unequal near PDI Check monocular acuity, a sidedness score was used with + values right eye better, values left eye better and 0 indicating bilaterally symmetric or equal acuity. A similar score was obtained for each monocular set of red, green and blue color tests subtracted left from right percent score so a positive value was better score right eye and a negative score was better score left eye. Multivariable analysis model predicting eye dominance highlighted an inverse impact of $\log _{10}$ stereo $(\mathrm{p}<0.01)$ and positive impact from average sided score from all three colors $(\mathrm{p}<0.01)$. Eye sidedness with near visual acuity and individual red, green and blue color sidedness had no significant impact in the predictive model for eye dominance. 
Table I Values for Stereo, Acuity and Color for Champion Shotgun Shooters and Normal Subjects. PDI Check Dynamic Autostereoscopic Near Vision Test Does Not Have a Threshold "Floor" for Highest Performance Stereo and Color Vision Unlike Titmus Fly. \# is the Number of Subjects Including 29 Shotgun Shooters with "Experienced" Meaning Those Classified as Expert or Advanced. The Non-Parametric Median with Interquartile Range (QI and Q3) and 95 Percentile Ranges are Compared to Mean and Standard Deviation (S.D.) Even Though Frequency Distributions Were Not Normal. logStereo is the Base-10 Log of PDI Check Stereo. VaR and VaL are Autostereoscopic Monocular Dynamic Landholt C Acuity. PDI Check Trichromatic Color for Red, Green and Blue Cones Either Right or Left ( $r$ or I) Eyes and Right Eye Also Compares Testing Clear and with Dark (Cherry) and Filtered (Yellow) Goggles

\begin{tabular}{|c|c|c|c|c|c|c|c|c|c|}
\hline & & \# & $5 \%$ & QI & Median & Q3 & $95 \%$ & Mean & S.D. \\
\hline \multirow[t]{3}{*}{ TitmusFly } & Shotgun & 29 & 40 & 40 & 40 & 50 & 100 & 71.4 & 140 \\
\hline & Experienced & 25 & 40 & 40 & 40 & 50 & 100 & 75.6 & $|5|$ \\
\hline & Army & 14 & 40 & 40 & 40 & 60 & 800 & 129 & 216 \\
\hline \multirow[t]{3}{*}{ PDI stereo } & Shotgun & 29 & 8.0 & 12.0 & 21.0 & 45.0 & 94.0 & 35.6 & 37.1 \\
\hline & Experienced & 25 & 9.0 & 12.0 & 17.0 & 41.0 & 94.0 & 34.1 & 38.6 \\
\hline & Army & 14 & 8.1 & 30.5 & 58.7 & 487 & 841.0 & 247 & 307 \\
\hline \multirow[t]{3}{*}{ logStereo } & Shotgun & 29 & 0.90 & 1.08 & 1.32 & 1.65 & 1.97 & 1.38 & 0.37 \\
\hline & Experienced & 25 & 0.95 & 1.08 & 1.23 & 1.61 & 1.97 & 1.36 & 0.36 \\
\hline & Army & 14 & 0.91 & 1.48 & 1.75 & 2.69 & 2.92 & 1.97 & 0.68 \\
\hline \multirow[t]{3}{*}{ VaR } & Shotgun & 29 & 20 & 20 & 20 & 20 & 40 & 22.5 & 8.7 \\
\hline & Experienced & 25 & 20 & 20 & 20 & 20 & 40 & 22.9 & 9.3 \\
\hline & Army & 14 & 20 & 20 & 30 & 40 & 40 & 30.0 & 14.1 \\
\hline \multirow[t]{3}{*}{$\mathrm{VaL}$} & Shotgun & 29 & 20 & 20 & 20 & 20 & 32 & 21.8 & 6.1 \\
\hline & Experienced & 25 & 20 & 20 & 20 & 20 & 32 & 21.9 & 6.5 \\
\hline & Army & 14 & 20 & 20 & 23 & 25 & 25 & 22.5 & 3.5 \\
\hline \multirow[t]{4}{*}{ Red-r } & Shotgun & 29 & 60.0 & 73.0 & 77.0 & 85.0 & 90.0 & 76.8 & 9.6 \\
\hline & Dark filter & 29 & 73.0 & 89.0 & 93.0 & 94.0 & 95.0 & 89.6 & 7.3 \\
\hline & Yellow filter & 29 & 68.0 & 77.0 & 80.0 & 88.0 & 91.0 & 79.7 & 10.2 \\
\hline & Color team & 12 & 52.0 & 65.0 & 72.0 & 75.0 & 84.0 & 70.3 & 9.4 \\
\hline \multirow[t]{2}{*}{ Red-I } & Shotgun & 29 & 43.0 & 64.0 & 73.0 & 83.0 & 87.0 & 72. & 13.9 \\
\hline & Color team & 12 & 35.0 & 55.0 & 61.5 & 69.0 & 73.0 & 59.7 & 11.4 \\
\hline \multirow[t]{4}{*}{ Green-r } & Shotgun & 29 & 59.0 & 84.0 & 90.0 & 95.0 & 99.0 & 86.8 & 12.3 \\
\hline & Dark filter & 29 & 81.0 & 94.0 & 98.0 & 99.0 & 100.0 & 95.6 & 6.2 \\
\hline & Yellow filter & 29 & 75.0 & 82.0 & 94.0 & 97.0 & 100.0 & 90.3 & 9.3 \\
\hline & Color team & 12 & 40.0 & 75.0 & 84.5 & 90.0 & 97.0 & 79.3 & 16.5 \\
\hline \multirow[t]{2}{*}{ Green-I } & Shotgun & 29 & 57.0 & 84.0 & 91.0 & 93.0 & 97.0 & 85.8 & 12.9 \\
\hline & Color team & 12 & 20.0 & 73.0 & 84.0 & 90.0 & 97.0 & 75.3 & 22.3 \\
\hline \multirow[t]{4}{*}{ Blue-r } & Shotgun & 29 & 66.0 & 85.0 & 88.0 & 92.0 & 95.0 & 86.6 & 7.5 \\
\hline & Dark filter & 29 & 51 & 83.0 & 91.0 & 92.0 & 99.0 & 85.4 & 15.5 \\
\hline & Yellow filter & 29 & 72 & 87.0 & 91.0 & 93.0 & 96.0 & 87.5 & 9.2 \\
\hline & Color team & 12 & 69.0 & 81.0 & 83.5 & 88.0 & 89.0 & 82.6 & 6.3 \\
\hline \multirow[t]{2}{*}{ Blue-I } & Shotgun & 29 & 74.0 & 83.0 & 88.0 & 90.0 & 94.0 & 85.1 & 8.8 \\
\hline & Color team & 12 & 75.0 & 84.0 & 88.0 & 90.0 & 93.0 & 85.6 & 5.9 \\
\hline
\end{tabular}

Note: Data Access: http://www.abcd-vision.org/references/PDI\%20shotgun\%200.2.15.pdf

Abbreviations: IQR, interquartile range between QI and Q3; logMAR, logarithm of the minimum angle of resolution; PDI Check, the name of an autostereoscopic vision testing game; S.D., standard deviation; log, logarithm; TNO, a brand of random dot stereogram; VaR and VaL are near visual acuity right and left.

\section{Discussion}

Using a novel near vision testing tool on the autostereoscopic barrier screen on the Nintendo 3DS console, we demonstrated superior dynamic near stereo and a trend toward better near dynamic trichromatic color in champion shotgun shooters compared to normal, age-matched Army 


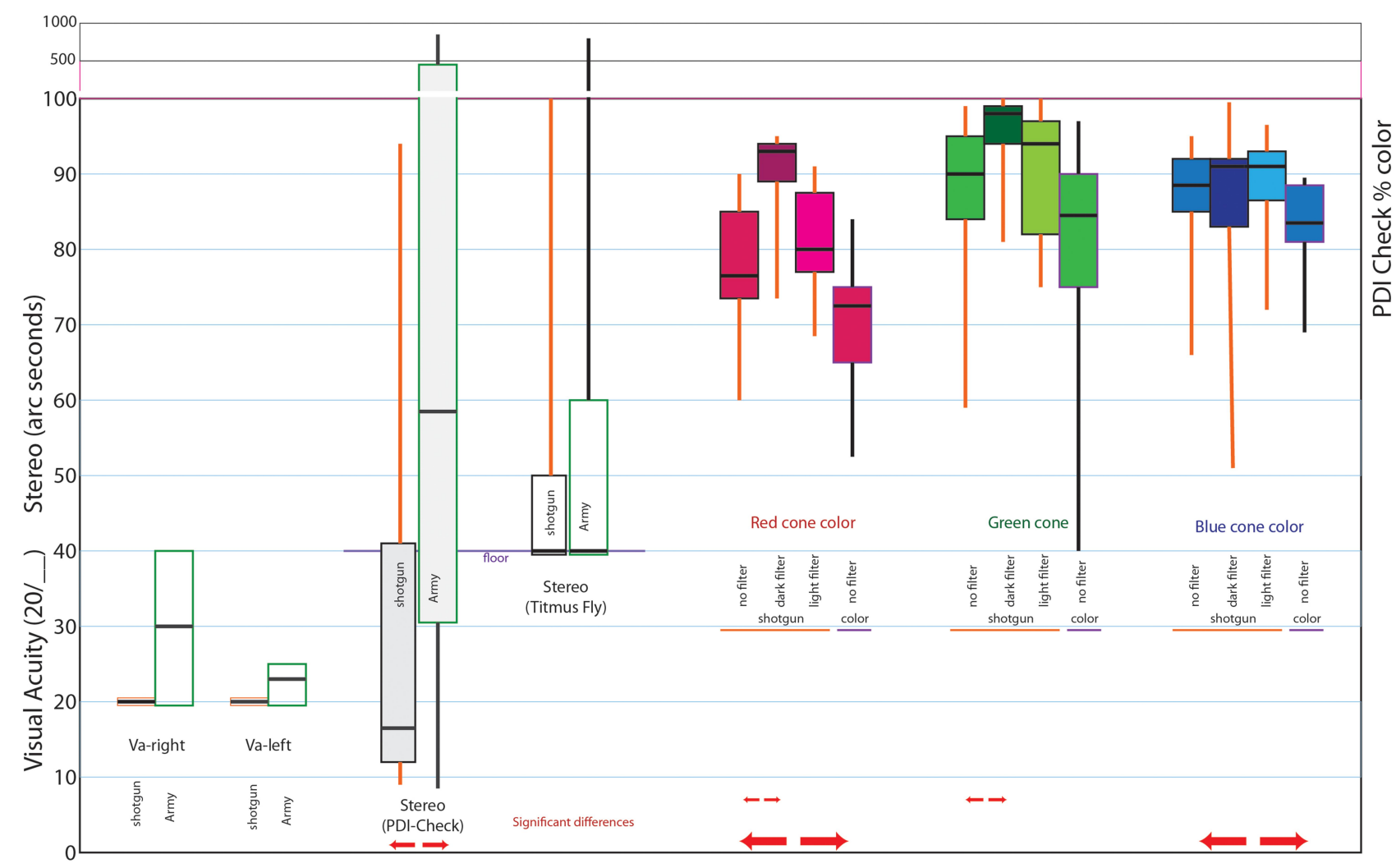

Figure I Box and whisker plots of near vision performance from PDI Check comparing champion shotgun shooters with normal Army volunteers. The vertical scale covers three variables; visual acuity as the 20/20 denominator calibrated near output from PDI Check, arc seconds of stereo (Titmus Fly and PDI Check) and percent color scores. Va-right and Va-left are monocular rivalry Landholt $C$ forced-choice dynamic recognition scores. Trichromatic dynamic monocular scores separate red cone, green cone and blue cone performance. Subjects included experienced shotgun shooters (orange whisker plots), age-matched Army volunteers (green boxes with black whiskers) and agematched Innova-Rabin passed normal volunteers (purple box with black whiskers). Shotgun shooters tested colors with and without dark and filtered goggles. Statistically different groups identified by red double arrows below.

and civilian volunteers. The Army volunteers previously had faster and better performance than villagers from a remote region in Burma. ${ }^{7}$ In another recent validation study, normal color-vision civilian volunteers demonstrated the rapid capacity for PDI Check to identify inherited protanomaly and deuteranomaly compared to Innova Rabin industry-standard computerized testing. ${ }^{9}$ The PDI Check scores did not definitively predict eye dominance with stereo or monocular performance in shotgun shooters with a sample size of 29 .

Separating exceptional visual function from ordinary function requires a test without a "floor effect" that limits the most precise presentation of stimuli. ${ }^{2}$ Computer distance visual acuity tests on high-resolution monitors are not limited to best presentation of logMAR $0(20 / 20)$ but can reliably present logMAR 0.6 (20/8). For conventional static stereo tests, the limitation on precision is $30 \mathrm{sec}$ arc. The static tests differ with respect to global or local presentation of perceived depth. Even the Innova Rabin computerized color test has a ceiling effect; it assesses red, green and blue cone hues calibrated as $100 \%$ with no ability to classify an extraordinary subject as better than $100 \%{ }^{11}$

PDI Check has evolved since 2017 initially with a version just testing stereopsis and then with subsequent versions testing color and near visual acuity on the Nintendo 3DS XL console. The upper, autostereoscopic Nintendo 3DS $124 \mathrm{~mm}$ wide screen has twin $400 \times 240$ pixel WQVGA resolution. Each Nintendo 3DS XL pixel measures $0.31 \mathrm{~mm}$ which compares to an iPhone with "retina display" having each pixel subtend $0.08 \mathrm{~mm}$. In order to screen for a visual qualitative sensation related to high-grade near visual acuity, the PDI Check vision testing paradigm uses dynamic presentation from supra-threshold precision (without floor effect) to gross presentation (with a theoretical ceiling effect) and favors forced 4-choice instead of forced two-choice method. ${ }^{12}$ Stereo is generated by gradually separating two rings- one right eye and the other left eye, with sub-pixel separation visually perceived by the round contour of the rings. The rivalry presentation on the autostereoscopic barrier screen allows screening for 
acuity and also suppression. ${ }^{6}$ Color can be tested in a dynamic, forced choice manner either with Farnsworth-Munsell type comparison, or trichromatic isoluminance monocular gray presentation. ${ }^{9}$ Former PDI Check color validation studies utilized Ishihara color plates as controls with few color deficient subjects and are much less rigorous since Ishihara is more of a screening test, and not a quantitative test of color. ${ }^{7}$

Abernethy and Neal compared expert to novice shotgun shooters on static and dynamic visual acuity, ocular muscle balance, ocular dominance, depth perception and color vision failing to demonstrate superior visual performance as a feature of exceptional shooting ${ }^{13}$ Shotgun shooting performance was neither improved, nor degraded by ingestion of a moderate amount of caffeine ${ }^{14}$ Vision in cricket and rugby players did not differ from normal other than slightly better TNO stereo in the elite cricket players. ${ }^{15}$

Shotgun coaches employ different methods to assess eye dominance; we queried each shooter as to their preferred eye. One method locates eye dominance anywhere along a spectrum between the two pupils with strong dominance near one pupil and weak dominance on a side but near the nose. $^{4}$ Further research will utilize this technique in an increased sample size of experienced shooters and using vision assessment without floor- or ceiling-effect.

Strengths of our study are uniform indoor testing with the same Nintendo 3DS XL console and the same version of the PDI Check software presenting stereo to the Army and shooters and trichromatic color for agematched normal subjects. The goggles used to compare color performance are actual shooter spectacles with the same pair used for all shooters. Weaknesses of the study included using historic controls in different environments and having a relatively small sample size of agematched normal subjects. We did not perform multiple test-retest analyses for shotgun shooters or for our control subjects.

\section{Conclusion}

Doctors seek tests that can quickly and reliably sort disease from healthy patients. Athletes and coaches seek tests, which can striate ordinary performance from extraordinary performance; tests without floor - and ceiling-effect are required. Shotgun shooters require quick, accurate detection of small, colored moving targets. The dynamic forced choice presentation on the PDI Check game has the potential to help doctors and coaches quickly discern monocular and binocular visual acuity, stereo and trichromatic color.

\section{Disclosure}

Drs Arnold and Smith are board members of PDI Check which makes vision screening games for autostereoscopic screens like the Nintendo 3DS. Dr. Arnold has a patent pending on PDI Check. Dr. Arnold is President and board member of Glacier Medical Software which markets ROP Check cloud-based NICU software. Dr. Arnold coordinates the Alaska Blind Child Discovery which has received discounted vision screening technology from several vendors. Dr. Arnold is the investigator and protocol developer for PEDIG. He also serves as the non-paid advisory board for PlusoptiX, Adaptica, and GoCheck Kids. Mr. Rue is an NSCA Level III Instructor. Stephen Brown is attending Martin Methodist University on a shotgun scholarship. The authors have no other conflicts of interest to report.

\section{References}

1. Kara S, Gencer B, Ersan I, et al. Repeatability of contrast sensitivity testing in patients with age-related macular degeneration, glaucoma, and cataract. Arq Bras Oftalmol. 2016;79(5):323-327. doi:10.5935/ 0004-2749.20160092

2. Coutant BE, Westheimer G. Population distribution of stereoscopic ability. Ophthalmic Physiol Opt. 1993;13(1):3-7. doi:10.1111/j.14751313.1993.tb00419.x

3. O'Connor AR, Tidbury LP. Stereopsis: are we assessing it in enough depth? Clin Exp Optom. 2018;101(4):485-494. doi:10.1111/cxo.12 655

4. Rue C. Kicking the bear. Clay target nation. National Skeet Shooting Association 5931 Roft Road, San Antonio, TX 78253-9261; 2019:64-66. Available from: www.nssa-nsca.org. Accessed January 29, 2021.

5. Smith KA, Damarjian AG, Molina A, Arnold RW. Calibrated measurement of acuity, color and stereopsis on a Nintendo 3DS game console. Clin Optom (Auckl). 2019;11:47-55. doi:10.2147/OPTO. S199992

6. Smith KA, Arnold AW, Sprano JH, Arnold SL, Arnold RW. Performance of a quick screening version of the Nintendo 3DS PDI check game in patients with ocular suppression. $J$ Pediatr Ophthalmol Strabismus. 2019;56(4):234-237. doi:10.3928/019139 13-20190502-01

7. Martin SJ, Rowe KS, Hser N, et al. Compared near vision testing with the Nintendo 3DS PDI check game on the Thai-Burma border. Asia Pac J Ophthalmol (Phila). 2019;8(4):330-334. doi:10.1097/ APO.0000000000000251

8. Holmes D. Eye Dominance Issues: What are the Options? Wiltshire England: Clay Shooting USA; 2015:34-41.

9. Arnold AW, Smith KA, Molina A, Damarjian AG, Arnold RW. Trichromatic enhanced dynamic color screening on the PDI check Nintendo 3DS game. Clinical Optometry (submitted).

10. Vancleef K, Read JCA, Herbert W, Goodship N, Woodhouse M, Serrano-Pedraza I. Overestimation of stereo thresholds by the TNO stereotest is not due to global stereopsis. Ophthalmic Physiol Opt. 2017;37(4):507-520. doi:10.1111/opo.12371 
11. Rabin J, Gooch J, Ivan D. Rapid quantification of color vision: the cone contrast test. Invest Ophthalmol Vis Sci. 2011;52(2):816-820. doi:10.1167/iovs.10-6283

12. Vancleef K, Read JCA, Herbert W, Goodship N, Woodhouse M, Serrano-Pedraza I. Two choices good, four choices better: for measuring stereoacuity in children, a four-alternative forced-choice paradigm is more efficient than two. PLoS One. 2018;13(7):e0201366. doi:10.1371/journal.pone.0201366
13. Abernethy B, Neal RJ. Visual characteristics of clay target shooters J Sci Med Sport. 1999;2(1):1-19. doi:10.1016/S1440-2440(99)80180-7

14. Share B, Sanders N, Kemp J. Caffeine and performance in clay target shooting. J Sports Sci. 2009;27(6):661-666. doi:10.1080/026404109 02741068

15. Barrett BT, Flavell JC, Bennett SJ, et al. Vision and visual history in elite/near-elite-level cricketers and rugby-league players. Sports Med Open. 2017;3(1):39. doi:10.1186/s40798-017-0106-Z
Clinical Ophthalmology

\section{Publish your work in this journal}

Clinical Ophthalmology is an international, peer-reviewed journal covering all subspecialties within ophthalmology. Key topics include: Optometry; Visual science; Pharmacology and drug therapy in eye diseases; Basic Sciences; Primary and Secondary eye care; Patient Safety and Quality of Care Improvements. This journal is indexed on PubMed

Submit your manuscript here: https://www.dovepress.com/clinical-ophthalmology-journal
Dovepress

Central and CAS, and is the official journal of The Society of Clinical Ophthalmology (SCO). The manuscript management system is completely online and includes a very quick and fair peer-review system, which is all easy to use. Visit http://www.dovepress.com/ testimonials.php to read real quotes from published authors. 